\title{
Psychometric Properties of the Arabic Version of the Functional Assessment of Chronic Illnesses Therapy-Fatigue (FACIT-F) in Arabic Cancer Patients
}

Mohammed Al Maqbali ${ }^{\mathrm{a}}$, Ciara Hughes ${ }^{\mathrm{a}}$, Jackie Gracey ${ }^{\mathrm{a}}$, Jane Rankin ${ }^{\mathrm{b}}$, Eileen Hacker ${ }^{\mathrm{c}}$, Lynn Dunwoody $^{\mathrm{d}}$

a Institute of Nursing and Health Research , Ulster University, Newtownabbey, UK

b Physiotherapy Department, Cancer Centre, Belfast Health and Social Care Trust, Belfast, UK

c School of Nursing, Indiana University, Indianapolis, USA

d Psychology Research Institute , Ulster University, Coleraine, UK

\begin{abstract}
:
Background: The Functional Assessment of Chronic Illness Therapy (FACIT) is a measurement system that was developed to assess the health-related quality of life among patients with cancer and other chronic illnesses. The Functional Assessment of Chronic Illness Therapy- Fatigue (FACIT-F) is a 40-item questionnaire, and it is the one of most frequently used instrument to assess fatigue in cancer populations. The aim of the study is to evaluate the psychometric properties of the Arabic FACIT-F among patients diagnosed with cancer.

Methods: Following a translated and cross-cultural evaluation procedure, of the FACIT-F Arabic version. A cross-sectional and descriptive correlational design was conducted. A total of 369 patients with cancer completed the FACIT-F, which consists of the Functional Assessment of Cancer Therapy - general scale's (FACT-G) 27-items, and the 13-item Functional Assessment of Chronic Illness Therapy-Fatigue (FACIT-Fatigue). The scale was assessed in terms of acceptability, internal consistency and validity. Construct validity was explored through confirmatory factor analysis.
\end{abstract}

Results: The FACT-G had acceptable fit in the four factor model, whereas the FACIT-Fatigue was found to be acceptable for the one-factor model in Arabic patients diagnosed with cancer. The Cronbach's alpha coefficient for the Arabic FACIT-Fatigue was 0.92, whereas the total score for FACT-G was 0.92, which showed good reliability. There was evidence that discriminate validity analysis was generally very good for the FACIT-Fatigue and FACT-G Arabic versions.

This is the author's manuscript of the article published in final edited form as:

Al Maqbali, M., Hughes, C., Gracey, J., Rankin, J., Hacker, E., \& Dunwoody, L. (2020). Psychometric Properties of the Arabic Version of the Functional Assessment of Chronic Illnesses Therapy-Fatigue in Arabic Cancer Patients. Journal of Pain and Symptom Management, 59(1), 130-138.e2. https://doi.org/10.1016/j.jpainsymman.2019.10.008 
Conclusion: The Arabic versions of the FACIT-Fatigue and FACT-G demonstrated good reliability and validity for assessing fatigue and quality of life in patients diagnosed with cancer.

Key Words: fatigue; FACIT-F; validity; Reliability; Arabic cancer patients

\section{Introduction:}

The diagnosis of cancer is a major life stressor that can affect the physiological, psychological and physical state of the individual. Cancer Related Fatigue (CRF) prevalence ranges between 50\% and $90 \% .{ }^{1}$ The majority of patients with cancer report fatigue as the most distressing symptom affecting their quality of life (QoL). ${ }^{2,3}$

Several instruments have been developed to measure CRF in English; one of the most widely used and validated is the Functional Assessment of Chronic Illnesses Therapy-F (FACIT-F). ${ }^{4,5}$ This questionnaire consists of QoL questions and an additional scale for measuring fatigue. ${ }^{6}$ The QoL is known as the Functional Assessment of Cancer Therapy - General (FACT-G). The FACT-G has four subscales: seven items for physical well-being (PWB); seven items for social/family wellbeing (SWB); six items for emotional well-being (EWB); and seven items for functional wellbeing (FWB). ${ }^{7}$ The additional scale for measuring fatigue, containing 13 items, is known as the Functional Assessment of Chronic Illnesses Therapy - Fatigue (FACIT-Fatigue), and can be used as a stand-alone brief assessment of fatigue. Therefore, FACIT-F refers to the 40-item questionnaire with all five subscales (physical, social/family, emotional functional, well-being and fatigue); FACT-G relates only to the four QoL subscales; and FACIT-Fatigue relates only to the 13-item fatigue scale.

FACIT-F 40-items has been validated for use with a variety of cancer diagnoses and treatments; the original scale showed strong internal consistency (coefficient alpha 0.93-0.95) and good stability (test-retest, $\mathrm{r}=0.87$ ). ${ }^{6}$ Other studies using the instrument have translated it into Spanish, ${ }^{8}$ French and Dutch ${ }^{9}$, Japanese, ${ }^{10}$ Persian, ${ }^{11}$ and Portuguese. ${ }^{12,13}$ The internal consistency in the translated versions ranged from 0.79 to 0.94 . 
A lack of appropriate scales to measure CRF is one of the challenges to identifying fatigue symptom disorders among patients with cancer in Arabic populations. Twenty-seven countries in the Middle East and North Africa have Arabic as the official language, and it is the 6th most spoken language in the world. ${ }^{14}$ However, the FACIT-F 40 -items has not been validated in clinical samples in Arabic speaking countries.

Therefore, the aim of this study was to examine the validity and reliability of the Arabic FACITF 40-items, version 4, in a heterogeneous sample of cancer patients in Oman. Validation of the Arabic FACIT-F 40-items in an Arabic country will provide a suitable and acceptable cultural fit instrument for screening and assessing fatigue in clinical practice.

\section{Methods:}

\section{Participants:}

A cross-sectional survey (November 2018 and January 2019) was used with participants recruited from the National Oncology Centre outpatients' clinic in Oman. The inclusion criteria were: ability to speak and write in Arabic, patients over 18 years of age, no known psychiatric or neurological disorders, diagnoses of any type of cancer, completed surgical intervention, chemotherapy or/and radiotherapy treatment at least one month prior to recruitment.

The sample size was determined as 10 participants required per item. ${ }^{15,16}$ For FACIT-Fatigue (13 items) 130 and FACT-G (27 items) 270 participants were required. Given the expectation of a nonresponders, a sample size of 400 participants was chosen.

Ethical permission was sought and granted by Research and Ethical Review and Approval Committee in the Directorate General of Planning and Studies at the Ministry of Health, Oman. Participants gave informed consent prior to completion of the survey.

\section{Instruments:}

Demographic information collected included age, gender, educational level, marital status, employment status, type of cancer, stage, type of treatment finished and time since diagnosis. Fatigue was measured using the FACIT-F. ${ }^{6}$ Responses were scored on a 5-point scale which ranged from 0 to 4 . 
The FACIT-Fatigue 13 items provided scores that ranged from 0 to 52 . Higher scores indicated no fatigue and the lowest score, the worst possible fatigue. The FACT-G was used to measure QoL, with the total score calculated by summing the 4 subscales. Possible scores ranged from 0 to 108 , with higher scores indicative of a good level of QoL. Permission was given by the developers to use the Arabic version of the FACIT-F instrument.

\section{Translation of FACIT-F:}

An Arabic version of the FACIT-F 40-items was available on the FACIT website (http://www.facit.org/TransHome) $)^{17}$ and translated using the method described by Bonomi et al.

${ }^{18}$ However, the FACIT team recommend cross-language and cross-cultural evaluation to test the instrument. ${ }^{19}$

To ensure the applicability of the instrument for the current study population, the FACIT-F 40items Arabic version was compared with the original English version by two independent bilingual translators. This ensured that the scales were translated into the Modern Standard Arabic language that is used for official purposes in all Arabic countries. The final version was compared with the original Arabic version proposed by the FACIT Group; it was evaluated by a team of three Arabic language experts, two translators and two oncology experts. To examine any difficulties in items comprehension of the FACIT-F, the scales were piloted with ten patients diagnosed with cancer. No changes for any words or phrases of the Arabic FACIT-F translation presented by the FACIT groups were necessary. [Online Supplement 1: FACIT-Fatigue 13-Items Arabic Version; Online Supplement 2: FACT-G 27-Items Arabic Version].

\section{Statistical Analyses:}

The 27-item FACT-G and 13-item FACIT-Fatigue were analysed separately. Construct validity of the FACT-G 27-items and FACT-F 13-items were examined using two separate Confirmatory Factor Analysis (CFA) conducted using AMOS 25 to test the hypothesis that the FACIT-Fatigue was unidimensional and the items in the FACT-G were best represented by a four-factor model. The following criteria were set to define acceptable model fit: Root Mean Square Error of Approximation (RMSEA) of less than or equal to 0.08; Comparative Fit Index (CFI) greater than 0.90; and Tucker-Lewis index (TLI) greater than 0.90. ${ }^{16}{ }^{20}$ Montan et al. ${ }^{21}$ 
previously used these model fit indices to examine the structure of the FACIT- Fatigue and FACTG.

The internal consistency of the two scales were evaluated using Cronbach's alpha coefficient for each of the subscales and the overall scales. Cronbach coefficient of 0.70 or higher was considered acceptable. ${ }^{22}$ The floor and ceiling effects were reported if more than $15 \%$ of the respondents achieved the lowest or highest possible scores $23 .^{22}$

Discriminant validity of the FACT-G and FACIT-Fatigue, determined by independent t-test and analysis of variance (ANOVA), is the ability of the scale to differentiate defined groups according to patient demographic and clinical characteristics. Statistical analyses were performed using the Statistical Package for the Social Sciences (SPSS) version 25.0. Significant levels of less than 0.05 were chosen, and all $p$ were two-sided.

\section{Results:}

Four hundred patients were eligible to participate; of these, 369 (92\%) returned valid questionnaires. Eleven refused participation, seven submitted incomplete questionnaires, and thirteen did not return the questionnaire.

The majority of the participants were female $66.4 \%(n=245) .28 \%$ had completed a secondary level education, $27.4 \%(\mathrm{n}=101)$ held a degree (college or higher); $20 \%$ of the participants had noformal education but they were able to read and write in Arabic (Table 1).

\section{Psychometric Properties}

\section{Confirmatory Factor Analysis}

The FACT-G had 34\% respondents did not answer GS7, due to participants exercising the option to skip GS7 (I am satisfied with my sex life). Factor analysis is sensitive to missing values and given that data were not missing randomly, imputation methods were deemed inappropriate. Hence, GS7 was removed from further CFA results for the 26-item FACT-G. This indicated that a four factor model was not an adequate fit for the data $\left(\lambda=0.22\right.$ to $0.85 ; \chi^{2} / \mathrm{df}=2.79, \mathrm{CFI}=0.88$;

RMSEA $=0.07$; TLI $=0.87$ ). Factor loadings for item GE2 (I am satisfied with how I am coping with my illness) were low $(\lambda=0.22)$; this item was removed from the second order factor model, 
which revealed a better fit $\left(\lambda=0.44\right.$ to $\left.0.88 ; \chi^{2} / \mathrm{df}=2.729, \mathrm{CFI}=0.90 ; \mathrm{RMSEA}=0.07 ; \mathrm{TLI}=0.90\right)$. A correlated error was apparent between items GF1 (I am able to work (including work at home)) and GF2 (My work (including work at home) is fulfilling); this resulted in an acceptable model fit $\left(\lambda=0.44\right.$ to $\left.0.88 ; \chi^{2} / \mathrm{df}=2.448, \mathrm{CFI}=0.92 ; \mathrm{RMSEA}=0.06 ; \mathrm{TLI}=0.91\right),($ Figure 1$)$.

A one-factor model ${ }^{24}$ was tested for the FACIT-Fatigue. Loadings for the 13 items ranged from $\lambda$ $=0.37$ to 0.89 , showing a less than optimal fit for the data $(\mathrm{CFI}=0.84 ; \mathrm{RMSEA}=0.15 ; \mathrm{TLI}=0.81)$. Modification indices were examined and six items were found to have significant shared error variance: An3 (I have trouble starting things because I am tired); An4 (I have trouble finishing things because I am tired); An5 (I have energy); An7 (I am able to do my usual activities); An15 (I am frustrated by being too tired to do the things I want to do) and An16 (I have to limit my social activity because I am tired). Items An3, An4, An15 and An16 had similar content. Items An5 and An7 were the only items in the FACIT-Fatigue -13 that were positively phrased, the other items were negatively constructed. The refined model fit indices of the sample were $\mathrm{CFI}=0.93$; RMSEA $=0.10 ;$ TLI $=0.91$, (Figure 2).

\section{Reliability:}

Internal consistency for the FACT-G 27-item scale was 0.92. Subscale values were 0.91 for PWB; 0.71 for SWB; 0.81 for EWB; and 0.87 for FWB. Internal consistency for the FACT-G 25-item scale (after removal of GS7 and GE2) was 0.92. Subscale values were 0.92 for PWB; 0.73 for SWB-6; 0.84 for EWB-5; and 0.87 for FWB.

The alpha coefficient of the fatigue scale for the FACIT-Fatigue 13-items Arabic version was 0.94. These findings indicated acceptable/excellent internal consistency for all subscales and aggregated scores, which compares favourably with those of the original English instrument. Ceiling and floor effect for scales and subscales were less than 15\%, other than SWB ceiling effect (17.6\%), due to the deleted item, GS7 (Table 2).

\section{Discriminant Validity:}

The mean scores of the FACIT-Fatigue, and the FACT-G and subscales showed discriminant validity between different patients' groups according to age, cancer site, stage of disease and comorbidities (Table 3). Fatigue scores were highest among patients with lung cancer compared 
to patients with other cancers. Cancer site was associated with significant differences in physical, emotional and functional well-being and total FACT-G score $(p<0.05)$. The mean score of the FACIT-Fatigue scale and FACT-G physical, social/family, emotional and functional well-being and total FACT-G score were able to discriminate between comorbidities versus no comorbidities $(p<0.05)$.

\section{Discussion:}

To our knowledge, this is the first psychometric study of the FACIT-Fatigue scale that has specifically been examined in an Arabic cancer population. The present study has shown that the FACT-G and FACIT-Fatigue scales reached acceptable levels of reliability and construct validity.

The internal consistency reliability of the Arabic version of the FACIT-Fatigue was $\alpha=0.94$, which was higher than the original study of Yellen et al. ${ }^{6}(\alpha=0.93)$. The internal consistency of the FACIT-Fatigue in Spanish was $0.88^{8}$, in Brazilian it was $0.79^{13}$, among patients diagnosed with almost similar cancers. The FACT-G subscales, PWB, SWB-6, EWB-5 and FWB, also had good reliability estimates ( 0.73 to 0.92 ) with total internal consistency of $\alpha=0.92$; this is in comparison with the result of the original version, which was $\alpha=0.89$.

Further, most of the FACIT-Fatigue and FACT-G scales and subscales did not have floor or ceiling effects: only SWB-6 presented with 17.5\%, and this may because of the removal of GS7. Therefore, items' analyses supports the content validity of the scale. ${ }^{23}$

Both the FACIT-Fatigue and FACT-G showed good to excellent evidence of construct validity; both scales appeared to be capable of discriminating between groups that differed in prognosis. The result of this study supports the discriminant validity of both scales. Previous research has established the discriminant validity of the FACT-G ${ }^{24}$ and the FACIT-Fatigue ${ }^{6}$, and has shown similar results in the original validation. Moreover, the finding of the discriminant validity of the Arabic version is consistent with Persian ${ }^{11}$ and English versions. ${ }^{25}$

Confirmatory factor analysis was conducted for the FACT-G and FACIT-Fatigue for all participants. The FACT-G had the highest rate of missing data; this was due to 125 (33.9\%) participants not answering GS7. In Arabic culture, sex is considered a private issue that cannot be discussed. ${ }^{26}$ This may explain the reluctance of participants to complete this item within the 
questionnaire. This is similar in different populations; for example Korean ${ }^{27}$ Chinese ${ }^{28}$ Spanish ${ }^{29}$ and Western European ${ }^{18}$, however, all those studies recommended to keep the GS7 item.

Item GE2 (I am satisfied with how I am coping with my illness) presented a problem with regards to the psychometric sensitivity as the item $(\lambda=0.22)$, had a low factor loading. This may be explained as cross-culturally problematic, as the word 'coping' translated into Arabic terms is 'Takuble'. In Islamic culture, Muslims' religious beliefs dictate that the disease or illness is God's will ${ }^{30}$; consequently, Muslims should cope with illness without regard to satisfaction. Another possible explanation was that GE2 is the only positively worded item among five items which were negatively worded. Previous studies have reported similar issues in Spanish ${ }^{29}$ and English ${ }^{25}$ version of FACT-G regarding the loading of GE2. This item may contribute to measurement error, ${ }^{16}$ and lead to a low factor loading.

In the final model, the correlation of items GF1 (I am able to work (including work at home)) and GF2 (My work (including work at home) is fulfilling) may be due to the similarity of items' content. A similar correlation was found between items GF1 and GF2 in the CFA conducted on cancer patients in Brazil. ${ }^{31}$ Although two items GE2 and GS7 were problematic to CFA, it recommended to keep the structure of FACT-G 27-items Arabic version the same as the original publication; also recommended by Dapueto et al. ${ }^{32}$, Lee et al. ${ }^{27}$ and Smith et al. ${ }^{33}$

The CFA of FACIT-Fatigue has been proposed as a unidimensional scale with the range of factor loadings being acceptable ( 0.39 to 0.89 ). The possible explanation for a high correlation between six items was that the participants had given similar answers: 59\% for An3 and An4, 48\% for An5 and An7, and 60\% for An15 and An16. This may indicate that these items have similar content.

Another possible reason for correlation between these items is due to the translation. In the English-Arabic comparison, for item An5 (I have energy), 'energy' is translated into Arabic as 'Alhayawia'; furthermore, in item An15, 'I am frustrated' was translated into Arabic as 'Asheur Bialaihbat'. These two words were translated into Modern Arabic and may have been misinterpreted by the participants. In this study, $44.4 \%$ of the participants had basic education or less; these participants may have had difficulty understanding these items. 
In this study, CFA confirmed the one factor structure of FACIT-Fatigue. Cella et al. ${ }^{34}$, Coroiu et al. ${ }^{35}$ and Montan et al. ${ }^{21}$ all showed that the 13-item FACIT-Fatigue scale has acceptable fit in the unidimensional model. This study found that RMSEA $=0.10$, which is below the set criterion $(\mathrm{RMSEA}=0.8)$ for an acceptable CFA model fit, however a similar result was reported by Cella et al. ${ }^{34}$ and Montan et al. ${ }^{21}$ It is recommended that the structure is kept and the contact instrument remain the same as the original publication so as not to violate the psychometric conditions for the translated instrument.

A think loud study would be recommended for FACIT-Fatigue and FACT-G Arabic versions in which the participant describes their thinking as they answer each items of the scales. ${ }^{36}$ This, method can provide content validity ${ }^{37}$ and response processes ${ }^{38}$ to each item of FACIT-Fatigue and FACT-G Arabic scales.

The current study has some limitations. The stability of the instrument was not reported using testretest reliability; therefore, further assessment of test-retest reliability is needed.

\section{Conclusion:}

This is the first study to validate the FACIT-F in a clinical sample in an Arabic speaking country. The Arabic versions of the FACIT-Fatigue and FACT-G, version four, have acceptable psychometric properties of internal consistency and discriminant validity. Therefore, the Arabic version of FACIT-F is a reliable and validated instrument to assess fatigue and QoL and can assess CRF in Arabic cancer populations. Further research on the Arabic versions should evaluate testretest reliability with a longitudinal methodology. 


\section{References:}

1. Campos MPO, Hassan BJ, Riechelmann R, Del Giglio A. Cancer-related fatigue: a practical review. Ann Oncol. 2011;22(6):1273-1279.

2. Cheng KKF, Wong WH, Koh C. Unmet needs mediate the relationship between symptoms and quality of life in breast cancer survivors. Support Care Cancer. 2016;24(5):2025-2033. doi:10.1007/s00520-015-2994-0

3. Iwase S, Kawaguchi T, Tokoro A, et al. Assessment of Cancer-Related Fatigue, Pain, and Quality of Life in Cancer Patients at Palliative Care Team Referral: A Multicenter Observational Study (JORTC PAL-09). PLOS ONE. 2015;10(8):e0134022. doi:10.1371/journal.pone.0134022

4. Seyidova-Khoshknabi D, Davis MP, Walsh D. Review article: a systematic review of cancer-related fatigue measurement questionnaires. Am J Hosp Palliat Care. 2011;28(2):119-129. doi:10.1177/1049909110381590

5. Al Maqbali M, Hughes C, Gracey J, Rankin J, Dunwoody L, Hacker E. Quality assessment criteria: psychometric properties of measurement tools for cancer related fatigue. Acta Oncol. 2019;58(9):1286-1297. doi:10.1080/0284186X.2019.1622773

6. Yellen SB, Cella DF, Webster K, Blendowski C, Kaplan E. Measuring fatigue and other anemia-related symptoms with the Functional Assessment of Cancer Therapy (FACT) measurement system. J Pain Symptom Manage. 1997;13(2):63-74. doi:10.1016/S08853924(96)00274-6

7. Webster K, Cella D, Yost K. The Functional Assessment of Chronic Illness Therapy (FACIT) Measurement System: properties, applications, and interpretation. Health Qual Life Outcomes. 2003;1(79):1-7. doi:10.1186/1477-7525-1-79

8. Dapueto JJ, del Carmen Abreu M, Francolino C, Levin R. Psychometric Assessment of the MSAS-SF and the FACIT-Fatigue Scale in Spanish-Speaking Patients With Cancer in Uruguay. J Pain Symptom Manage. 2014;47(5):936-945.

doi:10.1016/j.jpainsymman.2013.06.020 
9. Van Belle S, Paridaens R, Evers G, et al. Comparison of proposed diagnostic criteria with FACT-F and VAS for cancer-related fatigue: proposal for use as a screening tool. Support Care Cancer. 2005;13(4):246-254. doi:10.1007/s00520-004-0734-y

10. Yoshimura A, Kobayashi K, Fumimoto H, Fujiki Y, Eremenco S, Kudoh S. Cross-Cultural Validation of the Japanese Functional Assessment of Cancer Therapy-Anemia (FACT-An). J Nippon Med Sch. 2004;71(5):314-322. doi:10.1272/jnms.71.314

11. Meysami A, Yari H, Haghighat S, Montazeri A. The Functional Assessment of Chronic Illness Therapy - Fatigue (FACIT-Fatigue Subscale): Validity and Reliability of the Iranian Version. Oncol Res Treat. 2017;40(12):789-793. doi:10.1159/000479588

12. Ishikawa NM, Thuler LCS, Giglio AG, Baldotto CS da R, de Andrade CJC, Derchain SFM. Validation of the Portuguese version of Functional Assessment of Cancer Therapy-Fatigue (FACT-F) in Brazilian cancer patients. Support Care Cancer. 2010;18(4):481-490. doi:10.1007/s00520-009-0697-0

13. Ishikawa NM, Thuler LCS, Giglio AG, da Rocha Baldotto CS, de Andrade CJC, Derchain SFM. Reproducibility of Functional Assessment of Cancer Therapy Fatigue (FACT-F) questionnaire for cancer patients. Appl Cancer Res. 2008;28(2):55-61.

14. Horesh U, Cotter WM. Current Research on Linguistic Variation in the Arabic-Speaking World. Lang Linguist Compass. 2016;10(8):370-381.

15. Nunnally JC. Psychometric Theory. 2nd edition. New York: Mcgraw-Hill College; 1978.

16. Hair JF, Black WC, Babin BJ, Anderson RE. Multivariate Data Analysis: Pearson New International Edition. 7th ed. London: Pearson Higher Ed; 2014.

17. FACIT.org. Functional Assessment of Chronic Illness Therapy (FACIT) Measurement System. https://www.facit.org/facitorg/questionnaires. Published 2019. Accessed September 18, 2019.

18. Bonomi AE, Cella DF, Hahn EA, et al. Multilingual translation of the Functional Assessment of Cancer Therapy (FACT) quality of life measurement system. Qual Life Res. 1996;5(3):309-320. doi:10.1007/BF00433915

19. Eremenco SL, Cella D, Arnold BJ. A Comprehensive Method for the Translation and Cross-Cultural Validation of Health Status Questionnaires. Eval Health Prof. 2005;28(2):212-232. doi:10.1177/0163278705275342

20. Hu L, Bentler PM. Cutoff criteria for fit indexes in covariance structure analysis: Conventional criteria versus new alternatives. Struct Equ Model Multidiscip J. 1999;6(1):155. doi:10.1080/10705519909540118

21. Montan I, Löwe B, Cella D, Mehnert A, Hinz A. General Population Norms for the Functional Assessment of Chronic Illness Therapy (FACIT)-Fatigue Scale. Value Health. 2018;21(11):1313-1321. doi:10.1016/j.jval.2018.03.013 
22. McHorney CA, Tarlov AR. Individual-patient monitoring in clinical practice: are available health status surveys adequate? Qual Life Res. 1995;4(4):293-307.

doi:10.1007/BF01593882

23. Lim CR, Harris K, Dawson J, Beard DJ, Fitzpatrick R, Price AJ. Floor and ceiling effects in the OHS: an analysis of the NHS PROMs data set. BMJ Open. 2015;5(7):e007765. doi:10.1136/bmjopen-2015-007765

24. Cella DF, Tulsky DS, Gray G, et al. The Functional Assessment of Cancer Therapy scale: development and validation of the general measure. J Clin Oncol. 1993;11(3):570-579. doi:10.1200/JCO.1993.11.3.570

25. Eden MM, Kunkel K. Psychometric properties of the Modified Brief Fatigue Inventory and FACIT-Fatigue in individuals with cancer of the head and neck. Rehabil Oncol. 2016;34(3):97-103. doi:10.1097/01.REO.0000000000000024

26. Elobaid Y, Aw T-C, Lim JNW, Hamid S, Grivna M. Breast cancer presentation delays among Arab and national women in the UAE: a qualitative study. SSM - Popul Health. 2016;2:155-163. doi:10.1016/j.ssmph.2016.02.007

27. Lee E-H, Chun M, Kang S, Lee H-J. Validation of the Functional Assessment of Cancer Therapy-General (FACT-G) Scale for Measuring the Health-related Quality of Life in Korean Women with Breast Cancer. Jpn J Clin Oncol. 2004;34(7):393-399. doi:10.1093/jjco/hyh070

28. Zhou HJ, So JBY, Yong WP, et al. Validation of the functional assessment of cancer therapy-gastric module for the Chinese population. Health Qual Life Outcomes. 2012;10(145):1-8. doi:10.1186/1477-7525-10-145

29. Sánchez R, Ballesteros M, Arnold BJ. Validation of the FACT-G scale for evaluating quality of life in cancer patients in Colombia. Qual Life Res. 2011;20(1):19-29. doi:10.1007/s11136-010-9727-0

30. Boucher NA, Siddiqui EA, Koenig HG. Supporting Muslim Patients During Advanced Illness. Perm J. 2017;21. doi:10.7812/TPP/16-190

31. Campos JADB, Spexoto MCB, Serrano SV, Maroco J. Psychometric characteristics of the Functional Assessment of Cancer Therapy-General when applied to Brazilian cancer patients: a cross-cultural adaptation and validation. Health Qual Life Outcomes. 2016;14(1):8. doi:10.1186/s12955-015-0400-8

32. Dapueto JJ, Francolino C, Servente L, et al. Evaluation of the Functional Assessment of Cancer Therapy-General (FACT-G) Spanish Version 4 in South America: Classic Psychometric and Item Response Theory Analyses. Health Qual Life Outcomes. 2003;1(1):32. doi:10.1186/1477-7525-1-32 
33. Smith AB, Wright P, Selby PJ, Velikova G. A Rasch and factor analysis of the Functional Assessment of Cancer Therapy-General (FACT-G). Health Qual Life Outcomes. 2007;5:19. doi: $10.1186 / 1477-7525-5-19$

34. Cella D, Lai J-S, Stone A. Self-reported fatigue: one dimension or more? Lessons from the Functional Assessment of Chronic Illness Therapy-Fatigue (FACIT-F) questionnaire. Support Care Cancer. 2011;19(9):1441-1450. doi:10.1007/s00520-010-0971-1

35. Coroiu A, Kwakkenbos L, Levis B, et al. The Comparability of Functional Assessment of Chronic Illness Therapy - Fatigue Scores between Cancer and Systemic Sclerosis. $J$ Scleroderma Relat Disord. 2017;2(1):57-63. doi:10.5301/jsrd.5000227

36. Charters E. The Use of Think-aloud Methods in Qualitative Research An Introduction to Think-aloud Methods. Brock Educ J. 2003;12(2):68-82. doi:DOI.ORG/10.26522/BROCKED.V12I2.38

37. Peterson $\mathrm{CH}$, Peterson NA, Powell KG. Cognitive Interviewing for Item Development: Validity Evidence Based on Content and Response Processes. Meas Eval Couns Dev. 2017;50(4):217-223. doi:10.1080/07481756.2017.1339564

38. Castillo-Díaz M, Padilla J-L. How Cognitive Interviewing can Provide Validity Evidence of the Response Processes to Scale Items. Soc Indic Res. 2013;114(3):963-975. doi:10.1007/s11205-012-0184-8 



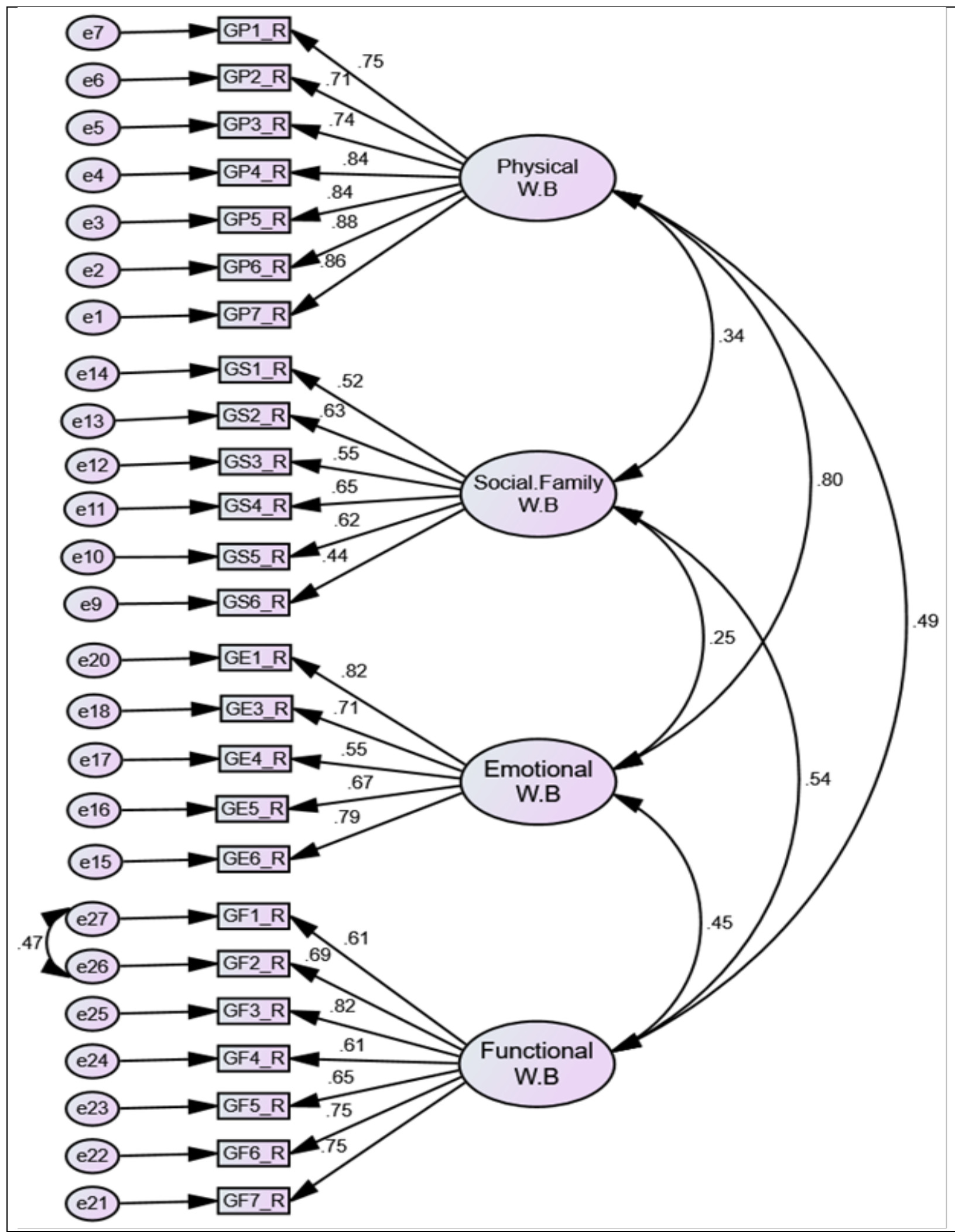

Figure 1: Factors structure of the Functional Assessment of Cancer Therapy-General 25-items $\lambda=.44$ to $.88 ; \chi 2 / \mathrm{df}=2.448$, Comparative Fit Index $(\mathrm{CFI})=.92$; Root mean Square Error of Approximation $($ RMSEA $)=.06$; Tucker-Lewis Index $(\mathrm{TLI})=.91$ 


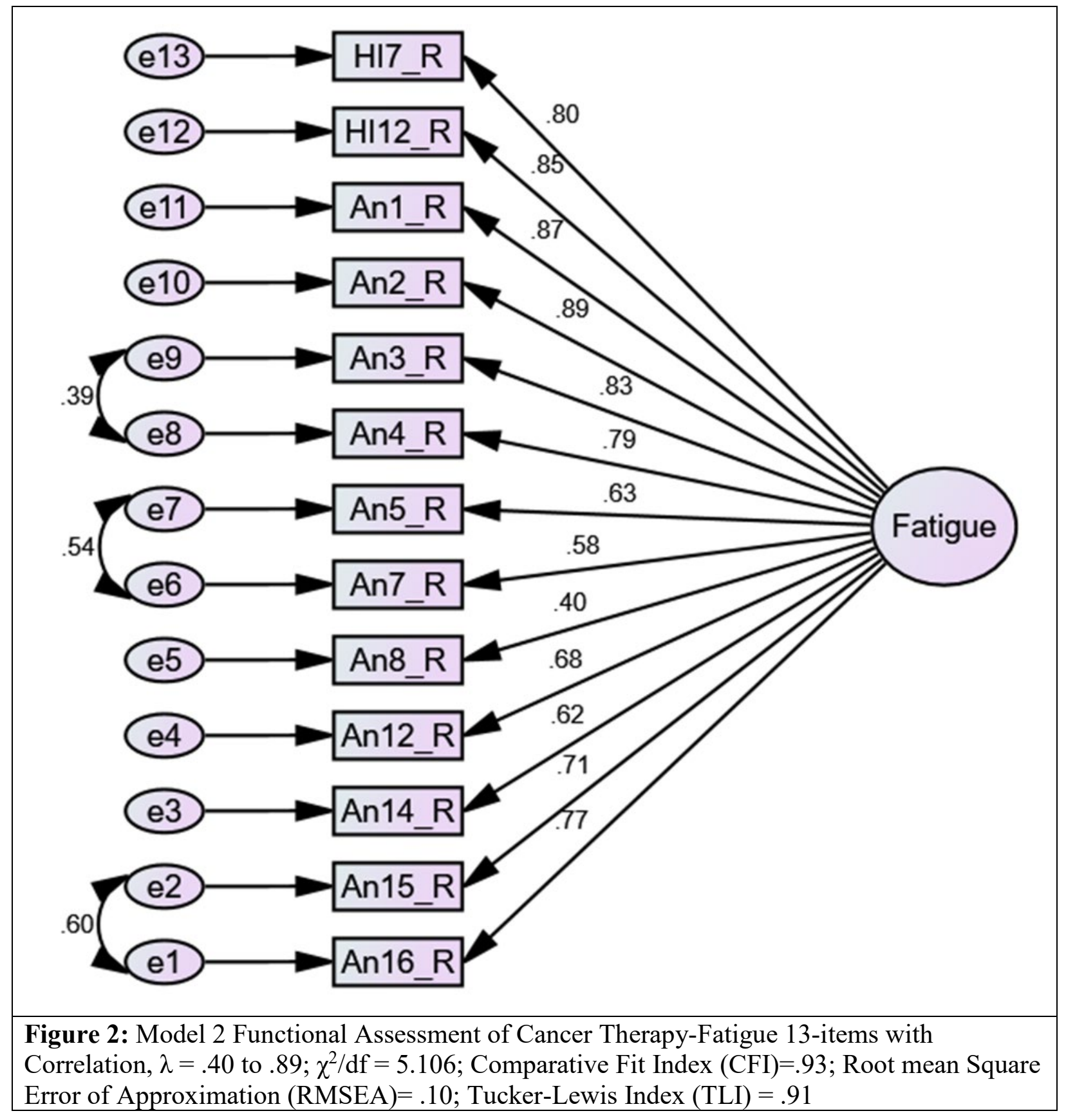

Table 1: Demographic and Clinical Characteristic of Participants ( $N=369)$ : 


\begin{tabular}{|c|c|c|}
\hline \multicolumn{3}{|c|}{ Demographic Characteristic } \\
\hline & $\mathrm{n}$ & $\%$ \\
\hline \multicolumn{3}{|l|}{ Gender } \\
\hline Male & 124 & 33.6 \\
\hline Female & 245 & 66.4 \\
\hline \multicolumn{3}{|l|}{ Age } \\
\hline $18-30$ & 36 & 9.8 \\
\hline $31-40$ & 84 & 22.8 \\
\hline $41-50$ & 95 & 25.7 \\
\hline $51-60$ & 76 & 20.6 \\
\hline More than 60 & 78 & 21.1 \\
\hline \multicolumn{3}{|l|}{ Marital Status } \\
\hline Married & 273 & 74 \\
\hline Single & 40 & 10.8 \\
\hline $\begin{array}{l}\text { Divorced/ Separated } \\
\text { /widowed }\end{array}$ & 56 & 15.2 \\
\hline \multicolumn{3}{|l|}{ Education Level } \\
\hline Non-Formal Education & 75 & 20.3 \\
\hline Basic Education & 89 & 24.1 \\
\hline Secondary Education & 104 & 28.2 \\
\hline Degree & 101 & 27.4 \\
\hline \multicolumn{3}{|l|}{ Employment Stats } \\
\hline Employed & 125 & 33.9 \\
\hline Retired & 71 & 19.2 \\
\hline Unemployed & 173 & 46.9 \\
\hline
\end{tabular}

\begin{tabular}{|lcc|}
\hline \multicolumn{3}{|c|}{ Clinical Characteristic } \\
\hline \multicolumn{2}{|c|}{$\mathrm{n}$} & $\%$ \\
\hline Cancer Site & & \\
\hline Breast & 139 & 37.7 \\
\hline Colorectal & 54 & 14.6 \\
\hline Stomach & 18 & 4.9 \\
\hline Others & 29 & 7.9 \\
\hline Lung & 20 & 5.4 \\
\hline Gastrointestinal & 19 & 5.1 \\
\hline Brain & 19 & 5.1 \\
\hline lymphoma & 20 & 5.4 \\
\hline Gynaecological & 24 & 6.5 \\
\hline Urinary & 27 & 7.3 \\
\hline Stage & & \\
\hline I & 34 & 9.2 \\
\hline II & 145 & 39.3 \\
\hline III & 107 & 29 \\
\hline IV & 72 & 19.5 \\
\hline Unspecified & 11 & 3 \\
\hline Month Since Diagnosis & & \\
\hline$<12$ months & 157 & 42.5 \\
\hline 12-24 months & 127 & 34.4 \\
\hline 25-36 months & 52 & 14.1 \\
\hline$>$ 36 months & 33 & 8.9 \\
\hline Type of Treatment & & \\
Finished & 13 & 3.5 \\
\hline Chemo and Radio & 234 & 63.4 \\
\hline Chemotherapy & 122 & 33.1 \\
\hline Radiotherapy & 3.5 \\
\hline Comorbidities & 53 \\
\hline None & 21 & 52.3 \\
\hline Asthma & 5.7 \\
\hline Anaemia & 16.5 \\
\hline Diabetes & 16.3 \\
\hline Hypertensive/ Heart & \\
Failure & & \\
\hline Diabetes/Heart diseases & 60 \\
\hline Others & & \\
\hline
\end{tabular}


Table 2: Internal Consistency Cronbach's $\alpha$ and Ceiling-Floor Effect for FACIT-F Version 4 (N=369)

\begin{tabular}{|c|c|c|c|c|c|c|c|}
\hline subscales & $\begin{array}{l}\text { Number of items } \\
\text { (Score Range) }\end{array}$ & Scores & & $\begin{array}{c}\text { Ceiling } \\
\text { effect }(\%)\end{array}$ & $\begin{array}{c}\text { Floor effect } \\
(\%)\end{array}$ & Cronbach's $\alpha$ & $\begin{array}{c}\text { Cronbach's } \alpha \\
\text { Original Version }\end{array}$ \\
\hline & & Mean & SD & & & & \\
\hline Physical Well-Being & $7(0-28)$ & 12.13 & 8.33 & $2.4 \%$ & $3.8 \%$ & .92 & $0.82^{a}$ \\
\hline $\begin{array}{r}\text { Social / Family Well- } \\
\text { Being }\end{array}$ & $6^{c}(0-24)$ & 19.75 & 3.82 & $17.6 \%$ & $.5 \%$ & .73 & $0.69^{a}$ \\
\hline Emotional Well-Being & $5^{d}(0-20)$ & 12.14 & 5.35 & $8.4 \%$ & $1.1 \%$ & .84 & $0.74^{a}$ \\
\hline Functional Well-Being & $7(0-28)$ & 19.28 & 5.79 & $7 \%$ & $.5 \%$ & .87 & $0.80^{a}$ \\
\hline FACT-G 25 items & $25(0-100)$ & 63.3 & 17.71 & $0.3 \%$ & - & .92 & $0.89^{a}$ \\
\hline FACT-Fatigue & $13(0-52)$ & 22.70 & 12.00 & $0.3 \%$ & $0.5 \%$ & .94 & $0.93^{b}$ \\
\hline FACIT-F & $38(0-52)$ & 86 & 29.15 & - & - & .95 & $0.95^{b}$ \\
\hline
\end{tabular}


Table 3: FACT-G and FACIT-Fatigue Scores According to the Patient Characteristics (Discriminant Validity):

\begin{tabular}{|c|c|c|c|c|c|c|c|c|c|c|c|c|}
\hline & \multicolumn{2}{|c|}{ FACIT-Fatigue } & \multicolumn{2}{|c|}{ FACT-G } & \multicolumn{2}{|c|}{ PWB } & \multicolumn{2}{|c|}{ SWB } & \multicolumn{2}{|c|}{ EWB } & \multicolumn{2}{|c|}{ FWB } \\
\hline & M(SD) & $P$ & M(SD) & $\boldsymbol{P}$ & M(SD) & $P$ & M(SD) & $P$ & M(SD) & $P$ & M(SD) & $P$ \\
\hline Age & & $<0.001$ & & $<0.001$ & & 0.001 & & $<0.001$ & & 0.007 & & $<0.001$ \\
\hline $18-30(36)$ & $25.3(12.9)$ & & $64.9(16.2)$ & & $13.4(7.9)$ & & $19.3(4.1)$ & & $11.9(5.4)$ & & $20.3(5.2)$ & \\
\hline $31-40(84)$ & $25.5(12.8)$ & & $69.3(17.5)$ & & $13.8(8.5)$ & & $20.9(3)$ & & $13.3(5.3)$ & & $21.2(5.0)$ & \\
\hline $41-50(95)$ & $25.3(12.9)$ & & $67.3(17.9)$ & & $13.3(8.5)$ & & $20.2(3.4)$ & & $13.1(5.5)$ & & $20.8(5.3)$ & \\
\hline $51-60(76)$ & $21.9(12)$ & & $60(16.1)$ & & $11.4(7.8)$ & & $19.6(4.1)$ & & $10.9(5.3)$ & & $18.1(5.3)$ & \\
\hline$>60(78)$ & $16.1(12)$ & & $54.4(15.9)$ & & $9(7.8)$ & & $18.3(4.3)$ & & $11(4.9)$ & & $16.1(6.4)$ & \\
\hline Cancer Site & & $<0.001$ & & $<0.001$ & & $<0.001$ & & 0.102 & & $<0.001$ & & $<0.001$ \\
\hline Breast (139) & $26.1(13.3)$ & & $68.2(17.4)$ & & $14.1(8.2)$ & & $20.4(3.4)$ & & $13.2(5.2)$ & & $20.5(5.1)$ & \\
\hline Colorectal (54) & $20.9(12.6)$ & & $61.3(16.9)$ & & $10.7(8)$ & & $20(3)$ & & $11.2(5.1)$ & & $19.5(5.6)$ & \\
\hline Stomach (18) & $23.8(11.8)$ & & $61.7(18.5)$ & & $13.1(9)$ & & $18.6(6.1)$ & & $12.6(5.9)$ & & $17.5(6.1)$ & \\
\hline Others (29) & $27.1(11.8)$ & & $69(15)$ & & $13.9(7.9)$ & & $20(4)$ & & $14.5(4.7)$ & & $20.6(5.2)$ & \\
\hline Lung (20) & $15(11.1)$ & & $50(13.7)$ & & $7.6(7.5)$ & & $17.7(5.4)$ & & $9.4(5)$ & & $15.3(7.8)$ & \\
\hline $\begin{array}{l}\text { Gastrointestinal } \\
\text { (19) }\end{array}$ & $17.2(12.3)$ & & $49.9(17.4)$ & & $7.4(8.6)$ & & 19.1(3.9) & & $9.8(4.5)$ & & $13.6(5)$ & \\
\hline Brain (19) & $15(10.9)$ & & $53.3(18.4)$ & & $7.7(8)$ & & $19.3(3.2)$ & & $8.6(6)$ & & $17.7(5.2)$ & \\
\hline Lymphoma (20) & $20.3(13.2)$ & & $63.9(15.2)$ & & $13.7(8.5)$ & & $18.7(4.6)$ & & $11.5(4.9)$ & & $20.1(5.3)$ & \\
\hline $\begin{array}{l}\text { Gynaecological } \\
(24)\end{array}$ & $20.6(10.1)$ & & $61(14.6)$ & & $10.4(6.7)$ & & $19.5(3.8)$ & & $12(5.1)$ & & $19.1(5.3)$ & \\
\hline Urinary (27) & $21.8(13.4)$ & & $65.1(18)$ & & $12.8(8.3)$ & & $19.8(3.5)$ & & $12.4(5.8)$ & & $20.1(6.4)$ & \\
\hline Stage of Disease & & 0.25 & & 0.004 & & 0.17 & & 0.11 & & 0.07 & & $<0.001$ \\
\hline I (34) & $25.1(13.1)$ & & $69.6(18.9)$ & & $14.1(8.1)$ & & $21.3(3.2)$ & & $13.4(5.8)$ & & $20.8(5.5)$ & \\
\hline II (145) & $23.6(11.9)$ & & $65.3(16.8)$ & & $12.8(8.4)$ & & $19.8(4.2)$ & & $12.7(5.2)$ & & $20(5.6)$ & \\
\hline III (107) & $22.7(13.7)$ & & $62.9(16.4)$ & & $12(8.1)$ & & $19.6(3.3)$ & & $12(5)$ & & $19.4(5.5)$ & \\
\hline IV (72) & $19.9(13.7)$ & & $57(19.3)$ & & $10.4(8.5)$ & & $19.2(3.7)$ & & $10.8(5.5)$ & & $16.6(6.2)$ & \\
\hline Unspecified (11) & $21.5(13.8)$ & & $62.5(17)$ & & $10.3(8.2)$ & & $19.6(5.1)$ & & $10.8(7)$ & & $21.8(3.5)$ & \\
\hline Comorbidities & & $<0.001$ & & $<0.001$ & & $<0.001$ & & .04 & & $<0.001$ & & $<0.001$ \\
\hline No (193) & $26.7(12.5)$ & & $68.6(16.8)$ & & $14.6(8.2)$ & & $22.9(4.5)$ & & $13.4(5.1)$ & & $20.6(5.6)$ & \\
\hline Yes (176) & $18.3(12.1)$ & & $57.5(16.8)$ & & $9.4(7.7)$ & & $21.9(4.5)$ & & $10.7(5.3)$ & & $17.9(5.6)$ & \\
\hline
\end{tabular}


